\title{
DNA damage induced by human CD40 ligand mutant promotes senescence and induces demethylation of GATA4 in lung cancer
}

\author{
YUE LI, YUNYAN WEI, WEIWEI YUAN, QIQING HUANG, YAYA ZHAO, \\ WEIHONG ZHAO, WEI XU and JIANQING WU \\ Jiangsu Provincial Key Laboratory of Geriatrics, Department of Geriatrics, \\ The First Affiliated Hospital of Nanjing Medical University, Nanjing, Jiangsu 210029, P.R. China
}

Received October 3, 2017; Accepted March 8, 2018

DOI: $10.3892 /$ or.2018.6310

\begin{abstract}
The ligand of CD40, known as CD154 or CD40L, is the key to immunostimulatory and anticancer activity, but how CD40L affects cellular senescence is unclear. Thus, we studied a membrane-stable mutant form CD40L (CD40L-M) to explore tumor growth and cellular senescence in CD40-positive NSCLC cells. We found that CD40L-M-expressing cells had senescent characteristics, including reduced cell proliferation and enlargement, increased SA- $\beta$-gal staining activity, and overexpression of several cell cycle regulators p53 and p21. In addition, expression of GATA4 was restored, and the $\mathrm{NF}-\kappa \mathrm{B}$ signaling pathway was activated in the CD40L-M-induced senescent cells. Mechanistic analyses revealed that $\mathrm{CD} 40 \mathrm{~L}-\mathrm{M}$ expression triggered the ATM/Chk2 DNA damage response, which mediated cell senescence and GATA4 activation. Knockdown of GATA4 reversed CD40L-M-induced senescence and decreased NF- $\kappa$ B activity. Thus, CD40L-M contributes to induction of cell senescence in CD40-positive NSCLC cells, and GATA4 is a switch to activate the NF- $\kappa \mathrm{B}$ pathway, which is positively regulated by DNA damage response (DDR) signaling kinases. Collectively, CD40L-M-induced senescence may be a barrier to the growth of lung cancer cells.
\end{abstract}

Correspondence to: Professor Jianqing $\mathrm{Wu}$ or Dr Wei $\mathrm{Xu}$, Department of Geriatrics, The First Affiliated Hospital of Nanjing Medical University, 300 Guangzhou Road, Nanjing, Jiangsu 210029, P.R. China

E-mail: jwunjmu@163.com

E-mail:ann_hsu@njmu.edu.cn

Abbreviations: NSCLC, non-small cell lung cancer; CD40L-M, CD40 ligand mutant; SA- $\beta$-gal, senescence-associated $\beta$-galactosidase; ATM, ataxia telangiectasia mutated; ATR, ATM-related kinase

Key words: CD40L mutant, senescence, GATA4, DNA damage response, NSCLC

\section{Introduction}

Lung cancer is the most common cause of cancer-related death, accounting for $\sim 1.6$ million premature deaths. The majority (80\%) of lung cancer cases are non-small cell lung cancer (NSCLC), which is associated with a poor 5-year patient survival $(1,2)$. NSCLC therapy has traditionally relied on chemotherapeutics but poor response results in rare remissions and the side-effects are significant $(3,4)$. Thus, novel therapeutic strategies to improve treatment responses are a focus of anticancer research.

Cellular senescence is an important defense mechanism that prevents cancer development. Multiple stimuli can trigger senescence, including telomere attrition, epigenetic alterations, activation of oncogenes, DNA damage and oxidative stress, which cause functional and morphological changes in cells $(5,6)$. Senescent cells have distinctive phenotypes and biomarkers, such as an enlarged flat morphology, enhanced senescence-associated $\beta$-galactosidase (SA- $\beta$-gal) activity, and a senescence-associated secretory phenotype (SASP). Additionally, increased p53 and p21 proteins and activation of DNA damage response (DDR) are universal features of senescent cells (7).

The ligand of CD40 (CD40L) is a type II membraneassociated glycoprotein and a member of the TNF gene family (8). CD40L helps regulate the immune response and suppresses tumor growth. CD40L transgene expression in CD40-positive malignant cells, such as breast or bladder cancer cells, was found to produce a direct growth-inhibitory effect via apoptosis $(9,10)$. However, membrane-stable CD40L can be cleaved by matrix metalloproteases (MMPs), releasing soluble fragment sCD40L which promotes various systemic inflammatory responses (11). To diminish the adverse effects of CD40L immunotherapy, we used a membrane-stable mutant form CD40L (CD40L-M) resistant to cleavage. Widespread expression of CD40 by carcinomas indicates that more research is required to explore the therapeutic benefits of CD40L-M.

In the present study, we evaluated the effect of CD40L-M expression on senescence in CD40-positive NSCLC cells as well as the molecular mechanisms underlying CD40L-M-induced 
cancer cell senescence regulation. Our data offer a better understanding of the multiple antitumor effects of CD40L-M in NSCLC.

\section{Materials and methods}

Cell culture and reagents. Human CD40-positive lung adenocarcinoma cell lines A549 and H460 and human bronchial 16HBE epithelial cell line were purchased from the Cell Resource Center (Shanghai Institutes for Biological Sciences, Shanghai, China). The cisplatin-resistant A549 (A549/DDP) and paclitaxel-resistant A549 (A549/TR) cell lines were kindly provided by Professor Zhou at the Shanghai Pulmonary Hospital. All cell lines were maintained at $37^{\circ} \mathrm{C}$ in a $5 \% \mathrm{CO}_{2}$ atmosphere in Dulbecco's modified Eagle's medium (DMEM) containing 10\% fetal bovine serum (FBS; ScienCell Research Laboratories, San Diego, CA, USA), $100 \mathrm{U} / \mathrm{ml}$ penicillin and $100 \mu \mathrm{g} / \mathrm{ml}$ streptomycin. To maintain drug resistance, A549/DDP and A549/TR cells were grown in DMEM containing $6.67 \mu \mathrm{M}$ cisplatin or $0.23 \mu \mathrm{M}$ paclitaxel (Taxol $^{\circledR}$ ) (Sigma-Aldrich; Merck KGaA, Darmstadt, Germany) respectively, and then in drug-free DMEM two days before the experiments. We previously constructed a plasmid expressing CD40L-M, which contained 6 substitutions $\left(\mathrm{Gln}^{114}\right.$ to $\operatorname{Pro}^{114}$, Lys $^{115}$ to $\mathrm{Arg}^{115}$, Asp ${ }^{117}$ to $\mathrm{Glu}^{117}, \mathrm{Gln}^{118}$ to $\mathrm{Glu}^{118}$, Asn ${ }^{119}$ to $\mathrm{Asp}^{119}$ and $\mathrm{Pro}^{120}$ to $\mathrm{Ser}^{120}$ ) by Invitrogen Biotechnology (Shanghai, China). A549/TR, A549/DDP, H460 and A549 cells were transfected with an entry vector pcDNA3.1+, pcDNA3.1+-CD40L-WT or pcDNA3.1+-CD40L-M according to a previously described method (12). The ATM inhibitor KU-55933 was obtained from Selleck Chemicals (Houston, TX, USA).

siRNA transfection. To knockdown GATA4, cells were transfected with GATA4 siRNAs using Invitrogen Lipofectamine 2000 transfection reagent (Thermo Fisher Scientific, Inc., Waltham, MA, USA) according to the manufacturer's protocol. The siRNAs targeting sequences are as follows: CGAUAUGUUUGACGACUUC.

$\beta$-galactosidase senescence assay. SA- $\beta$-gal activity was measured with a $\beta$-galactosidase staining kit (Beyotime Institute of Biotechnology, Shanghai, China) according to the manufacturer's protocol. Briefly, the cells were cultured after transfection for $48 \mathrm{~h}$. Then, the cells were washed twice with PBS and fixed with a 3.5\% paraformaldehyde solution for $15 \mathrm{~min}$ at room temperature. Cells were washed every 5 min 3 times in PBS, and the SA- $\beta$-gal staining solution was added and incubated in a $37^{\circ} \mathrm{C}$ water bath for $16 \mathrm{~h}$. Images of the representative fields observed under a light microscope (Olympus IX-71; Olympus, Tokyo, Japan) were captured under 20x magnification.

Cell proliferation assay. Cell viability was measured using a Cell Counting Kit-8 (Bimake, Houston, TX, USA). Briefly, $5 \times 10^{3}$ cells were plated into each well of 96-well flat-bottomed plates. After $24 \mathrm{~h}$, the cells were transfected with an empty vector pcDNA3.1+, pcDNA3.1+-CD40L-WT or pcDNA3.1+-CD40L-M. Then cells were cultured for an additional $48 \mathrm{~h}$. A colorimetric assay was performed after addition of $10 \mu \mathrm{l}$ Cell Counting Kit- 8 reagent to each well, and plates were incubated at $37^{\circ} \mathrm{C}$ for $2-4 \mathrm{~h}$. Absorbance at $450 \mathrm{~nm}$ was read using a multiplate reader (Tecan Group Ltd., Männedorf, Switzerland).

Cell cycle analysis. For cell cycle analysis, $2 \times 10^{6}$ cells were harvested, fixed with $3 \mathrm{ml}$ of cold $75 \%$ ethanol at $-20^{\circ} \mathrm{C}$ overnight, and washed twice with PBS. The cells were then resuspended in $500 \mu \mathrm{l}$ of PBS and simultaneously stained with $200 \mu \mathrm{l}$ of DNA staining solution (MultiSciences, Hangzhou, China) at $25^{\circ} \mathrm{C}$ for $30 \mathrm{~min}$. The percentage of cells in each cell cycle phase was determined using a FACStation (FV500; Beckman Coulter, La Brea, CA, USA) and analyzed using Kaluza Flow Analysis software (Beckman Coulter, Inc.).

Immunofluorescence. Transfected cells were seeded on a micro-cover glass for $48 \mathrm{~h}$. Cells were fixed with $4 \%$ paraformaldehyde for $15 \mathrm{~min}$ and permeabilized with $0.3 \%$ Triton X-100 in PBS for $1 \mathrm{~h}$ at room temperature. After treatment, the slides were incubated with anti-GATA4 rabbit polyclonal antibody (1:100; cat. no. ab84593; Abcam, Cambridge, MA, USA) at $4^{\circ} \mathrm{C}$. Cells were washed and then incubated with goat anti-rabbit IgG $(\mathrm{H}+\mathrm{L})$ highly cross-adsorbed secondary antibody Alexa Fluor ${ }^{\circledR} 488$ conjugate (1:500; cat. no. A-11034; Thermo Fisher Scientific) for $1 \mathrm{~h}$ at room temperature. All slides were counterstained with 4'-6-diamidino-2-phenylindole (DAPI). Photomicrographs were captured using a fluorescence microscope (Olympus IX-71; Olympus).

Western blot analysis. Forty-eight hours after transfection, the cells were lysed using ice-cold RIPA buffer containing a mixture of phosphatase and protease inhibitors. Lysates were then centrifuged at $4^{\circ} \mathrm{C}$, and the supernatant was collected. Protein was measured in supernatants using the BCA method. Equal amounts of proteins $(30 \mu \mathrm{g})$ were separated on 10-12\% SDS-polyacrylamide gels and then blocked with $5 \%$ non-fat dry milk diluted in Tris-buffered saline $0.1 \mathrm{M}$ added to $0.1 \%$ Tween-20 (TBST). Membranes were incubated overnight at $4^{\circ} \mathrm{C}$ with one of the specific antibodies. Phospho-NF- $\mathrm{B}$ p65 (Ser536) (93H1) rabbit monoclonal antibody (mAb; 1:1,000; cat. no. 3033), NF- $\mathrm{KB}$ p65 (D14E12) rabbit mAb (1:1,000; cat. no. 8245), IкB $\alpha$ (44D4) rabbit mAb (1:1,000; cat. no. 4812), p53 (DO-7) mouse mAb (1:1,000; cat. no. 48818), p21 Waf1/Cip1 (12D1) rabbit mAb (1:1,000; cat. no. 2947), phospho-Chk2 (Thr68) (C13C1) rabbit mAb (1:1,000; cat. no. 2197), Chk2 (D9C6) rabbit mAb (1:1,000; cat. no. 6334), phospho-Chk1 (Ser317) (D12H3) rabbit mAb (1:1,000; cat. no. 12302), Chk1 (2G1D5) mouse $\mathrm{mAb}(1: 1,000$; cat. no. 2360), GAPDH (14C10) rabbit $\mathrm{mAb}(1: 1,000$; cat. no. 2118), $\beta$-Tubulin (9F3) rabbit $\mathrm{mAb}$ $(1: 1,000$; cat. no. 2128 , ) and a HRP-conjugated secondary antibody (1:2,000; cat. no. 7075) were obtained from Cell Signaling Technology (Beverly, MA, USA). CD40L (1:1,000; cat. no. ab65854) and GATA4 (1:1,000; cat. no. ab84593) were obtained from Abcam (Cambridge, MA, USA). After washing three times in TBST, a horseradish peroxidase-conjugated goat anti-rabbit antibody was applied. Proteins were visualized using Pierce ECL reagent (Thermo Fisher Scientific).

Methylation-specific PCR. DNA from A549, A549/TR and $16 \mathrm{HBE}$ cells was treated with sodium bisulfite and purified 
using EZ DNA methylation kit (Zymo Research, Irvine, CA, USA). Methylation-specific PCR (MSP) was used to determine bisulfite-induced changes affecting unmethylated (U) and methylated (M) alleles. Each MSP reaction incorporated $100 \mathrm{ng}$ of bisulfite-treated DNA, $25 \mathrm{pM}$ of each primer, 100 pM dNTPs, 10X PCR buffer, and 1 U/ml JumpStart Red Taq Polymerase (Sigma-Aldrich; Merck KGaA) in a final reaction volume of $25 \mu \mathrm{l}$. Cycle conditions were as previously described (13). MSP products were separated on a $2 \%$ agarose gel and stained with ethidium bromide. MSP primer sequences for GATA4 were as follows: GATA-4-M-sense, 5-GTATAG TTTCGTAGTTTGCGTTTAGC-3 and GATA-4-M-antisense, 5-AACTCGCGACTCGAATCCCCG-3; GATA-4-U-sense, 5-TTTGTATAGT TTTGTAGTTTGTGTTTAGT-3 and GATA-4-U-antisense, 5-CCCAACTCACAACTCAAATCC CCA-3.

Statistical analyses. Results are expressed as means \pm SD. All statistical analyses were performed using SPSS for Windows v.16.0 (SPSS, Inc., Chicago, IL, USA). Continuous data were analyzed using an independent Student's t-test between two groups. $\mathrm{P}<0.05$ was considered to indicate a statistically significant result.

\section{Results}

Expression of CD4OL-M induces cellular senescence. To investigate the multiple effects of CD40L-M, we used pcDNA3.1+, pcDNA3.1+-CD40L-WT and pcDNA3.1+-CD40L-M to transfect CD40-positive NSCLC cell lines. CD40L expression was increased in the A549, A549/TR, A549/DDP and H460 cells $48 \mathrm{~h}$ after transfection (Fig. $1 \mathrm{~A}$ and $\mathrm{B}$ ). In addition, the culture cell size was enlarged in the A549/TR, A549/DDP and $\mathrm{H} 460$ cells, a trait associated with senescence. These data were confirmed with SA- $\beta$-gal staining (Fig. 1C and D), yet SA- $\beta$-gal staining was not observed in the A549 cells. Thus, CD40L-M expression is implicated in cellular senescence in various CD40-positive NSCLC cells.

CD4OL-M contributes to the inhibition of cell proliferation and cell cycle arrest. There may be several features and cell cycle regulators in senescence networks. After $48 \mathrm{~h}$ of culture, total CD40L-M/A549/TR cells were not significantly increased compared with the controls or negative groups. CCK- 8 assay data showed that CD40L-M expression significantly reduced cell proliferation (Fig. 2A).

To explore growth inhibition after CD40L-M upregulation, we evaluated the effect of CD40L-M on cell cycle progression. Flow cytometry data showed that the percentage of $\mathrm{S}$ phase cells was decreased and the percentage of G0/G1 phase cells was increased in the CD40L-M-expressed cell group compared with these percentages noted in the controls or negative cells (Fig. 2B and C), indicating that CD40L-M expression blocked cell cycle progression. Expression of cell cycle regulators p53 and p21 were determined using western blot analysis and data showed that p53 and p21 expression was significantly increased in the CD40L-M-upregulated cells (Fig. 2D).

ATM/Chk2 is necessary for CD4OL-M-induced senescence. DNA damage response (DDR) is a trait of multi-faceted senescent phenotypes. To identify whether DDR is activated, we assessed p-Chk2 and p-Chk1 in CD40L-induced senescent A549/TR cells. Data showed that only p-Chk2 was significantly induced $48 \mathrm{~h}$ after transfection (Fig. 3A and B), indicating that the ATM/Chk2 pathway was activated in response to CD40L-M-induced senescence. To address the functional role of the ATM/Chk2 pathway, we treated CD40L-M-transfected A549/TR cells with an inhibitor of ATM kinase, KU-55933. KU-55933 inhibited phosphorylation of the ATM target protein Chk2 (Fig. 3C and D) and decreased SA- $\beta$-gal activity (Fig. 3E and F; P>0.05, compared to controls), suggesting that ATM/Chk2 mediated CD40L-M-induced senescence.

GATA4 is restored in CD4OL-M-induced senescent cells. GATA4 is a zinc-finger transcription factor, critical for the development of organogenesis, proliferation, differentiation and apoptosis. Numerous studies have shown that silencing of the GATA4 gene by promoter methylation has been implicated in carcinogenesis of the lung $(13,14)$. To investigate whether silencing of GATA4 expression is related to promoter region methylation, we used methylation-specific PCR to determine the methylation status of A549, A549/TR and 16HBE cells (Fig. 4A). Data showed that GATA4 methylation occurred in the A549 and A549/TR cells. In contrast, we observed no aberrant promoter hypermethylation for the GATA4 gene in $16 \mathrm{HBE}$ cells.

Cellular senescence causes widespread changes in chromatin organization (15). Prior studies have shown that DNA methylation is reduced in response to DNA damage or cell senescence (16). Thus, we hypothesized thatCD40L-M-induced senescence might be accompanied by GATA4 demethylation. Thus, we assessed the expression of GATA4 in CD40L-M-induced senescent A549/TR cells by western blot analysis. GATA4 protein was significantly increased in the CD40L-M-expressed A549/TR cells compared with that noted in the controls or negative groups (Fig. 4B). These data were confirmed by immunofluorescence (Fig. 4C).

GATA4 regulates senescence dependent on DDR. To determine whether GATA4 demethylation responds to DDR activation, KU-55933 was used to block ATM/Chk2 DDR. When the ATM/Chk2 pathway was suppressed, GATA4 expression was reduced in the CD40L-M-induced senescent cells (Fig. 5A and B).

To investigate whether GATA4 is associated with cellular senescence in CD40L-M-expressed A549/TR cells, we knocked down GATA4 using GATA4 siRNAs. Data showed that GATA4 protein was significantly downregulated (Fig. 5C and D) and suppression of GATA4 in CD40L-M-expressed A549/TR cells decreased SA- $\beta$-gal activity (Fig. 5E and F; P>0.05, compared to controls). Thus, GATA4 was responsible for CD40L-M-induced senescence which was dependent on DDR activation.

GATA4 regulates the $N F-\kappa B$ signaling pathway. To investigate how GATA4 regulates cell senescence, we explored the signaling pathways implicated in senescence. We measured phosphorylated NF- $\mathrm{B}$ p65 (p-p65) and its negative regulator I $\kappa \mathrm{B} \alpha$ using western blot analysis in the CD40L-M-induced senescent 

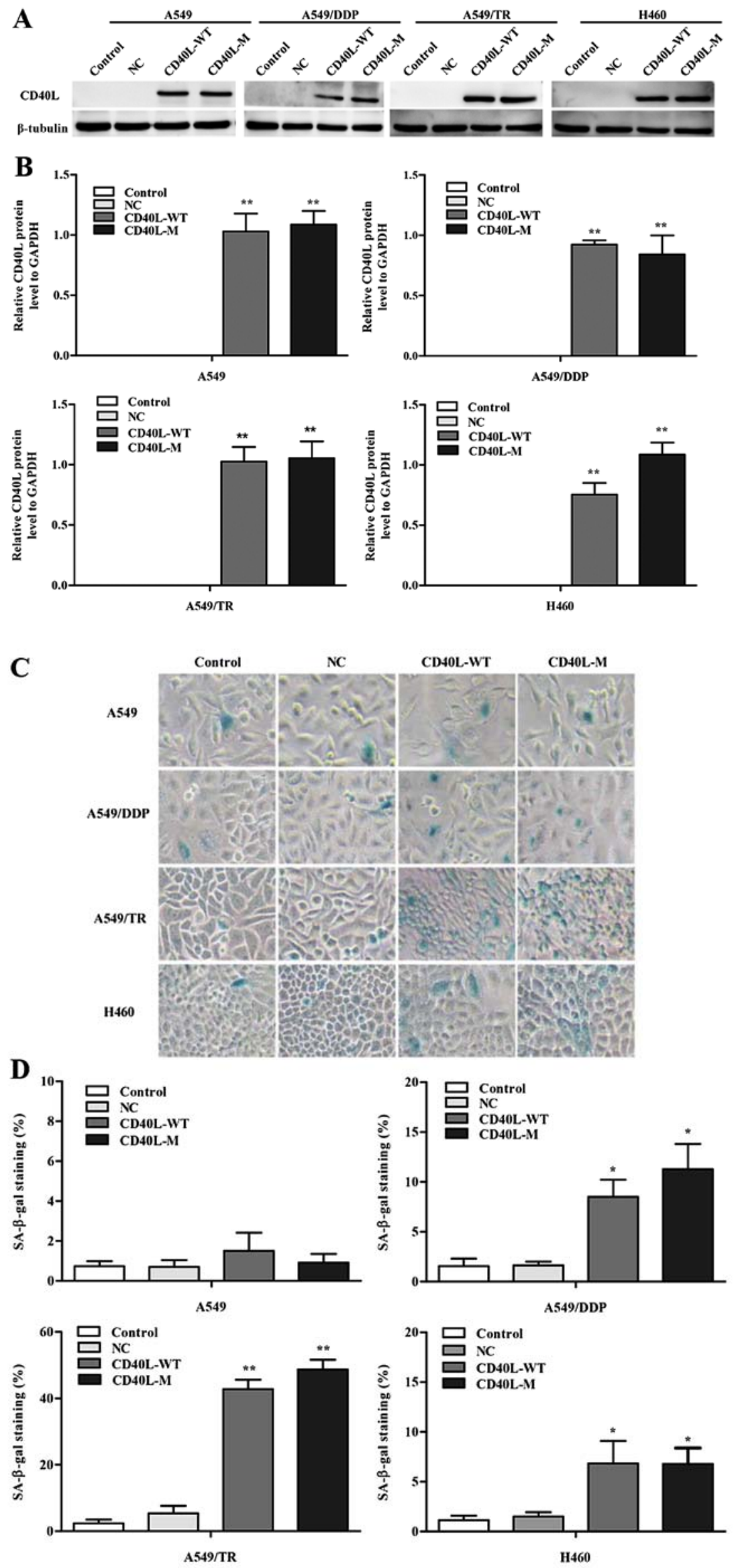

Figure 1. Expression of CD40L-M induces cellular senescence. (A) CD40-positive NSCLC A549, A549/TR, A549/DDP and H460 cell lines were transfected as indicated and $\mathrm{CD} 40 \mathrm{~L}$ protein was measured using western blot analysis and (B) quantified with densitometry, respectively. $\beta$-tubulin was used as a loading control. (C) Representative imageas of SA- $\beta$-gal staining and (D) the percentage of SA- $\beta$-gal-positive cells. Data are expressed as the means \pm SD of three independent experiments. ${ }^{*} \mathrm{P}<0.05,{ }^{* *} \mathrm{P}<0.01$ compared to the controls or negative group. 

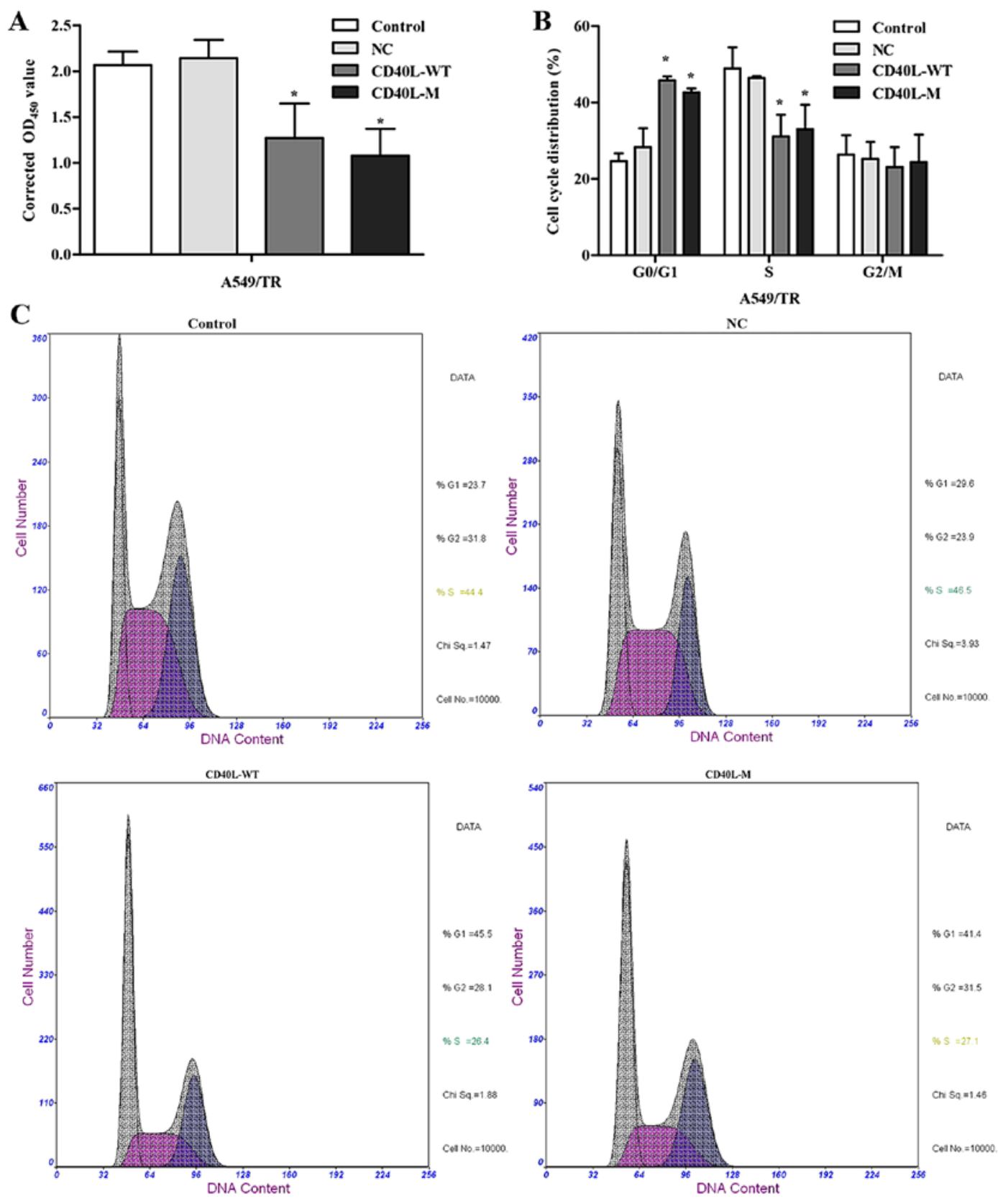

D
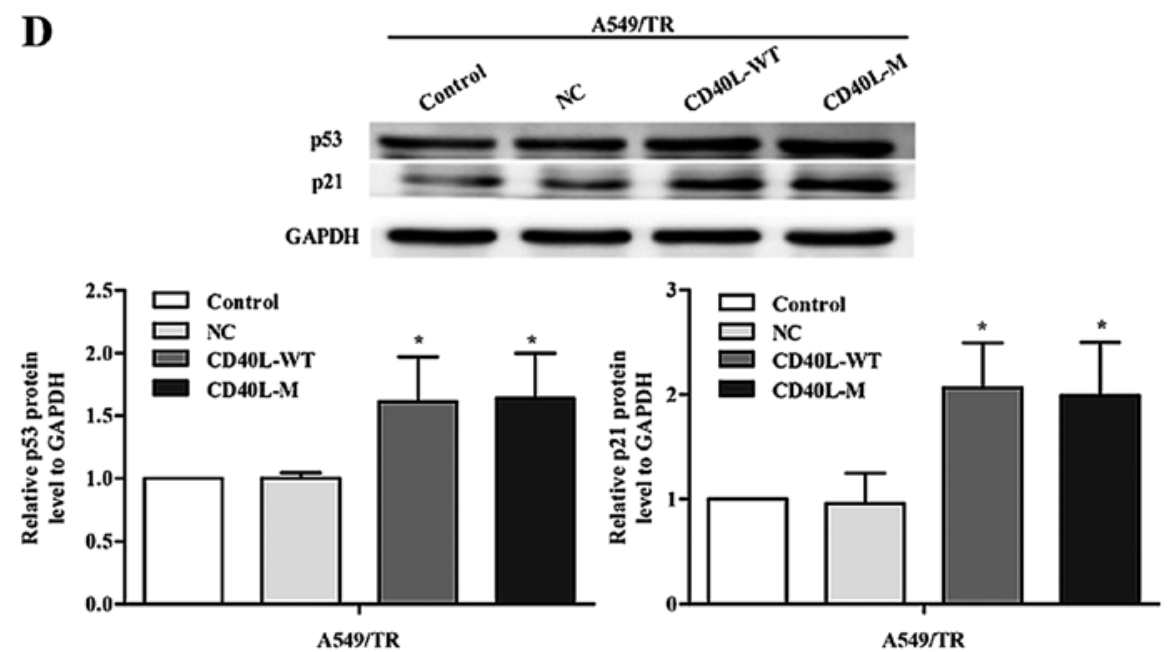

Figure 2. CD40L-M contributes to inhibition of cell proliferation and cell cycle arrest. (A) Cell proliferation was measured using a CCK-8 assay. (B) Cell cycle distribution. (C) The cell cycle was analyzed with PI staining and FACS. (D) p53 and p21 protein was determined using western blot analysis. GAPDH was used as a loading control. Data are expressed as the means \pm SD of three independent experiments. " $\mathrm{P}<0.05$ compared to the controls or negative group. 


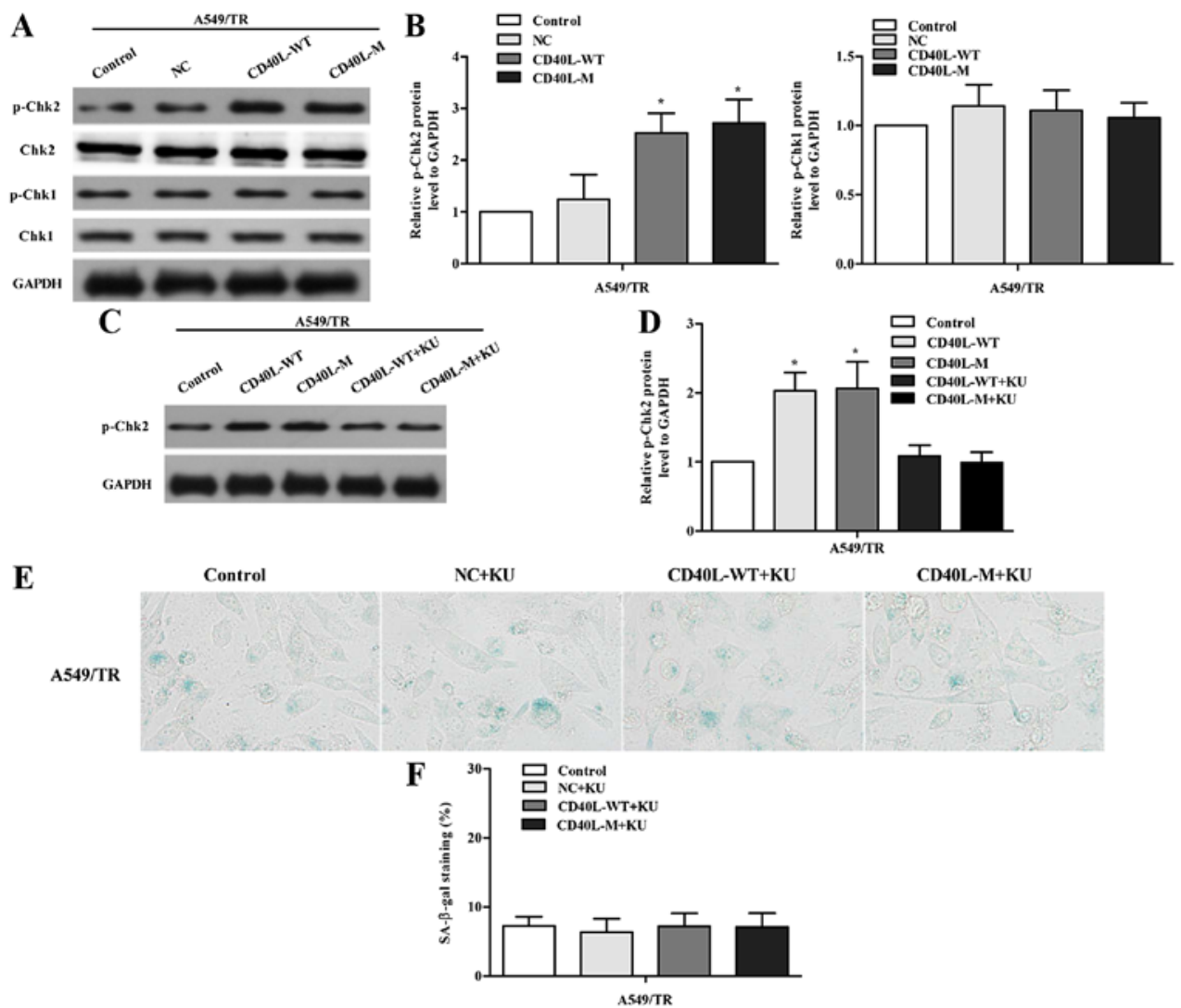

Figure 3. ATM/Chk2 is necessary for CD40L-M-induced senescence. (A) p-Chk1 and p-Chk2 expression was determined in CD40L-M-transfected cells by western blot analysis. (B) Quantitative analysis of protein levels. (C) p-Chk2 expression was assessed in ATM-specific inhibitor KU-55933-treated cells and (D) quantified by western blot analysis normalized to GAPDH. (E) SA- $\beta$-gal staining and (F) the percentage of SA- $\beta$-gal-positive cells. Data are expressed as the means \pm SD of three independent experiments. ${ }^{*} \mathrm{P}<0.05$ compared to the controls or negative group. KU, KU-55933.

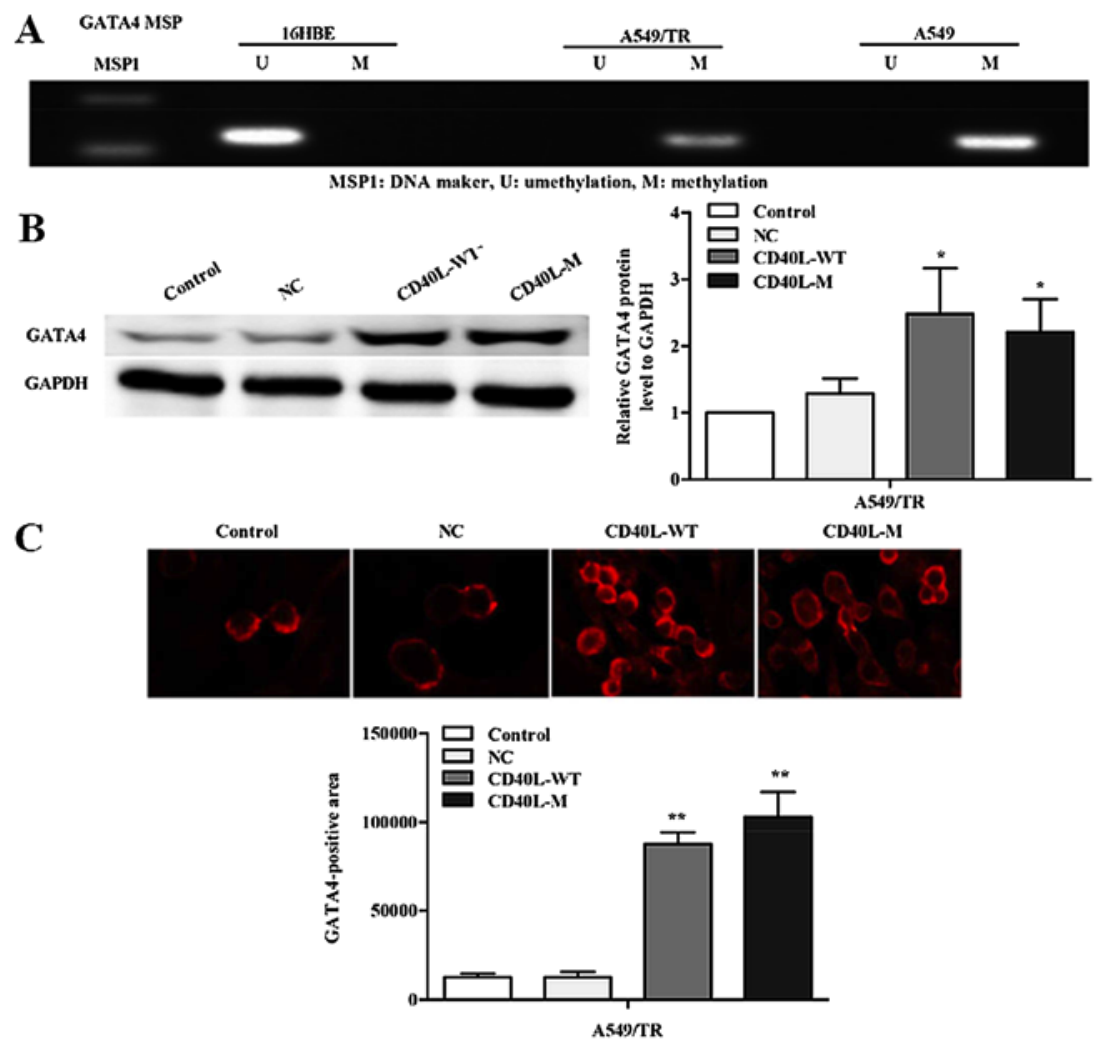

Figure 4. GATA4 is restored in CD40L-M-induced senescent cells. (A) Methylation-specific PCR for the GATA4 gene in A549, A549/TR and 16HBE cells. U represents amplification of unmethylated alleles; M represents methylated alleles. (B) GATA4 protein was measured using western blot analysis. (C) GATA4 expression was evaluated immunocytochemically. Data are expressed as the means $\pm \mathrm{SD}$ of three independent experiments. ${ }^{*} \mathrm{P}<0.05,{ }^{* * *} \mathrm{P}<0.01$ compared to the controls or negative group. 

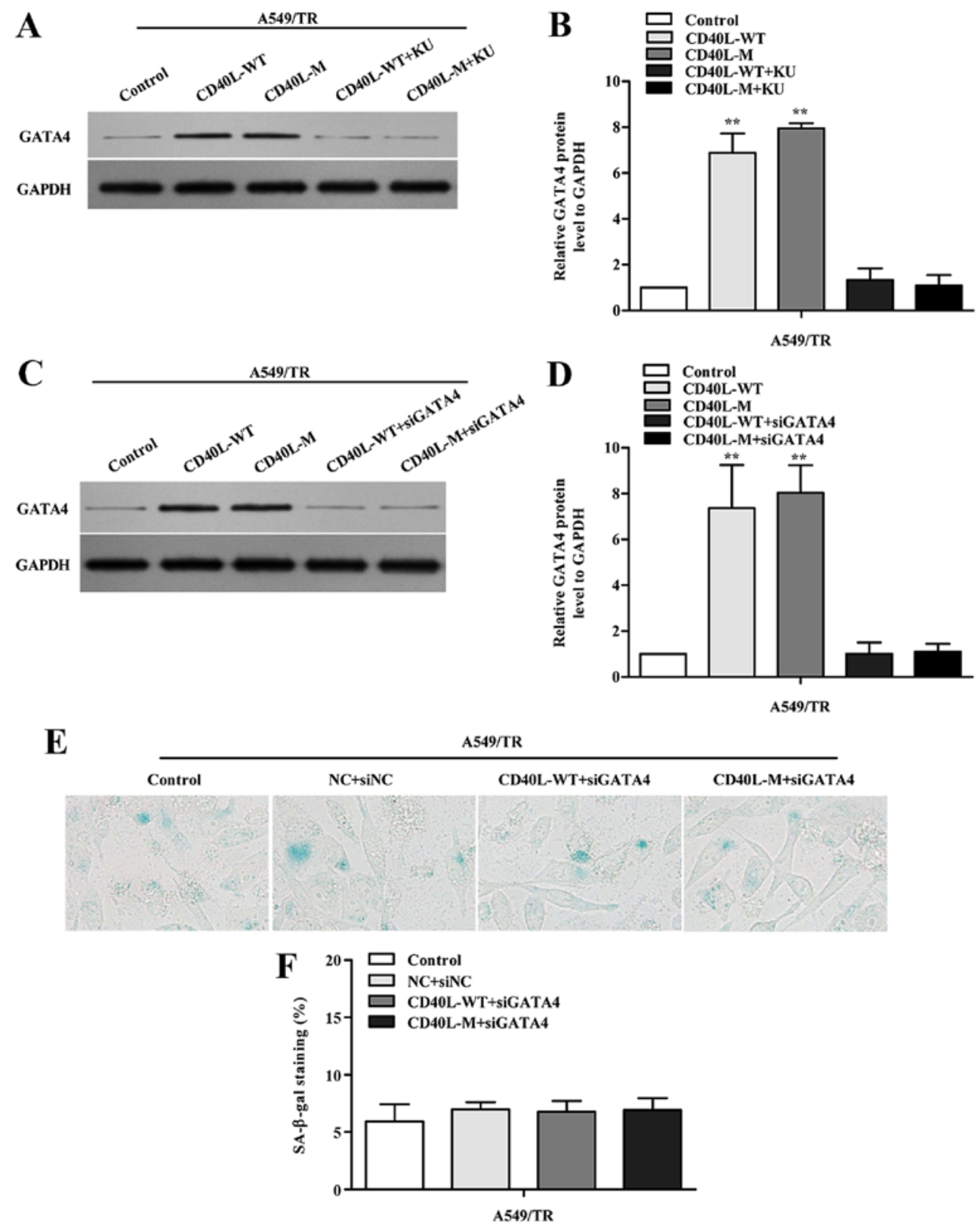

Figure 5. GATA4 regulates senescence dependent on DNA damage. (A) GATA4 was determined in CD40L-M-transfected A549/TR cells treated with KU-55933 using western blot analysis and (B) quantified with densitometry. (C) CD40L-M-upregulated A549/TR cells were co-transfected with siRNAs against GATA4 or corresponding negative control. GATA4 expression was evaluated by western blot analysis, and (D) quantitative protein analysis is shown. (E) Representative images of cells stained for SA- $\beta$-gal, and (F) the percentage of SA- $\beta$-gal-positive cells was analyzed. Data are expressed as the means \pm SD of three independent experiments. ${ }^{* *} \mathrm{P}<0.01$ compared to the controls or negative group. KU, KU-55933.

A549/TR cells and we found that expression of p-p65 was increased and $\mathrm{I} \kappa \mathrm{B} \alpha$ was reduced in the $\mathrm{CD} 40 \mathrm{~L}-\mathrm{M}$-expressing A549/TR cells (Fig. 6A and B). To confirm our results, we knocked down GATA4 with GATA4 siRNAs and found that p-p65 was partially decreased (Fig. 6C and D). Thus, the $\mathrm{NF}-\kappa \mathrm{B}$ signaling pathway was regulated by GATA4 in our cell systems.

\section{Discussion}

We reported that CD40L-M overexpression is associated with induction of senescence in CD40-positive NSCLC A549/TR, H460 and A549/DDP cell lines, whereas A549 cells displayed no senescent phenotype after CD40L-M upregulation.
In accordance with our previous studies, treatment with scAAV5 CD40L-M resulted in the significant reduction in cell number in the CD40 positive A549 cells by inducing apoptosis (17). In contrast to H460 and A549/DDP cells, more CD40L-M-expressed A549/TR cells exhibited an enlarged, flattened morphology accompanied by an increase in SA- $\beta$-gal staining activity. This might be attributed to the possibilities that A549/TR cells are inclined to senesce due to the DNA damage by Taxol previously, or A549/TR cells are more vulnerable to CD40L-induced senescence. Additionally, CD40L-M upregulation decreased cell proliferation and induced cell cycle arrest. p53 and its target gene p21 are essential regulators for cell cycle arrest after induction of senescence in response to DNA damage signals (18). We also showed that p53 and 

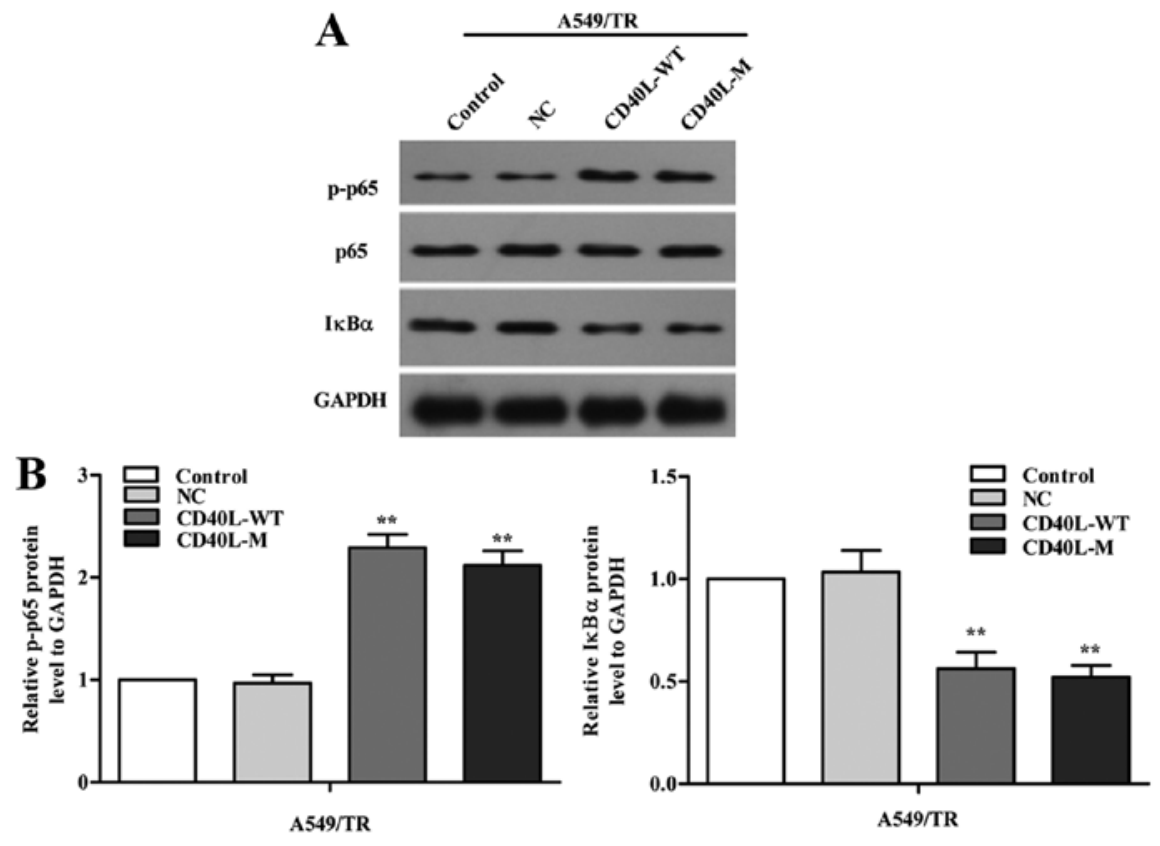

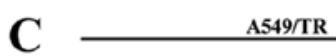

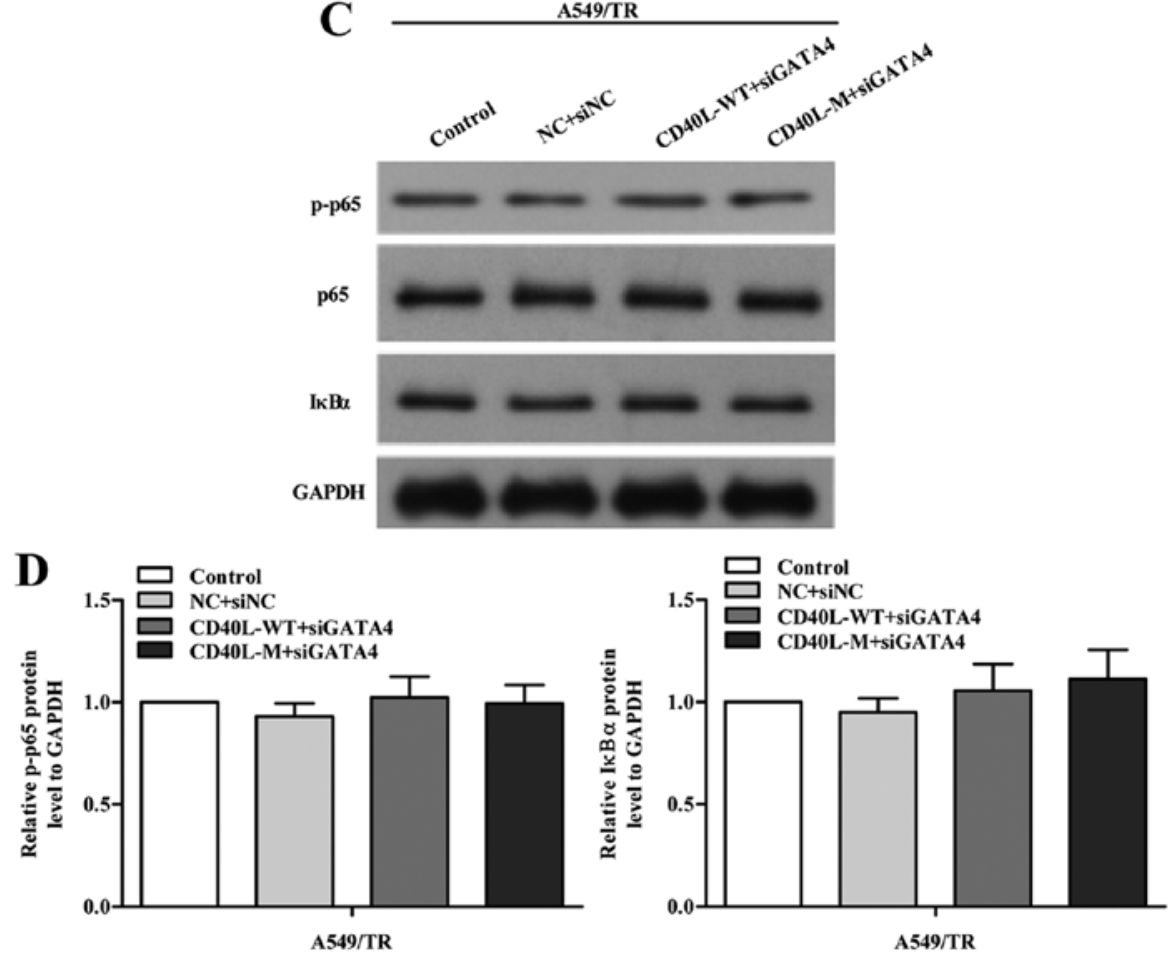

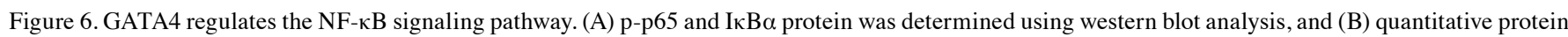
analysis is shown. (C) p-p65 and IкB $\alpha$ expression was confirmed in the GATA4 siRNA-transfected cells using western blot analysis. (D) Densitometric analysis of protein bands normalized to GAPDH. Data are expressed as the means \pm SD of three independent experiments. ${ }^{* *} \mathrm{P}<0.01$ compared to the controls or negative group.

p21 protein increased in the CD40L-M-induced senescent A549/TR cells. Thus, CD40L-M is involved in induction of cellular senescence in CD40-positive NSCLC cells with cell heterogeneity.

CD40L a key costimulatory molecule for antigenpresenting cells (APCs), which is preferentially expressed on activated $\mathrm{CD} 4^{+} \mathrm{T}$ cells and activated platelets $(19,20)$. Signaling from CD40L binding to its receptor CD40 induces antigen-presenting cells to express various immune accessory molecules and activates transcription factors, such as AP-1 and $\mathrm{NF}-\kappa \mathrm{B}$, which are crucial for the development of humoral and cellular immunity $(21,22)$. In addition, various forms of CD40L have been used to directly promote pro-apoptotic induction in CD40-positive malignant cells $(23,24)$. However, membrane-stable CD40L can be cleaved by various MMPs into a soluble form that induces survival signals in CD40-positive carcinomas causing inflammatory diseases $(11,25)$. To optimize CD40L gene therapy, we generated a membrane-stable 
mutant form CD40L resistant to MMPs. Our previous research showed that CD40L-M conferred a direct antitumor effect in vitro and in vivo with few side-effects $(12,17)$.

DNA damage response (DDR) is a senescent biomarker (26) and senescence-inducing stimuli can cause genomic damage, subsequently activating DDR (27). Our results showed that the ATM/Chk2 pathway was activated in CD40L-M-induced senescent NSCLC cells. Previous reports have shown that ATM or ATR activation is sufficient to induce cellular senescence $(28,29)$. Chk 2 can promote cellular senescence through either p53/p21 or other pathways (30). Therefore, we investigated whether Chk2 upregulation influences the regulation of cellular senescence in this context. Our data agree with these previous studies. We showed that $\mathrm{p}-\mathrm{Chk} 2$ suppression impaired cell senescence when we used an ATM inhibitor to block the ATM/Chk2 pathway. Thus, CD40L-M-induced senescence may be mediated by ATM/Chk2.

GATA4 is a transcription protein family member and common to other GATA factors, GATA4 contains two highly conserved zinc fingers that mediate DNA binding, and many protein interactions. GATA4 is frequently silenced by promoter methylation in lung, colorectal, prostate, ovarian, and breast cancers $(13,14)$. In contrast to tumor and surrounding normal tissue, the GATA4 promoter is either non-methylated or hypomethylated in healthy lung tissue (31). Consistent with these studies, our results showed that hypermethylation of GATA4 was determined in NSCLC A549/TR and A549 cell lines but not in 16HBE cells. Epigenomic perturbations are an inducer of cell senescence in response to various stimuli (32). Previous research has shown that epigenomic perturbations can activate DDR signaling (27). In contrast, our results showed that DDR contributed to GATA4 demethylation in senescent A549/TR cells expressing CD40L-M. A recent study showed that GATA4 is a key regulator of senescent phenotypes (33) and our data showed that GATA4 knockdown decreased SA- $\beta$-gal activity. Therefore, GATA4 expression was induced and positively regulated senescence in CD40L-M-upregulated A549/TR cells.

NF- $\kappa \mathrm{B}$ can be activated by diverse external and internal stimuli associated with senescence, such as DNA damage and genotoxic stresses (34). Because the NF- $\mathrm{B}$ signaling pathway can promote cellular senescence (35), we investigated the relationship between GATA4 and the NF- $\mathrm{B}$ pathway during CD40L-M-induced senescence in NSCLC cells. Data showed that the NF- $\mathrm{B}$ pathway was activated in the CD40L-M-overexpressed A549/TR cells. In addition, knockdown of GATA4 resulted in markedly reduced $\mathrm{NF}-\kappa \mathrm{B}$ activity. In fact, it has been clearly established that $\mathrm{NF}-\kappa \mathrm{B}$ positively regulates the senescence-associated secretory phenotype (SASP) that is a prominent property of senescent cells. Some SASP factors can reinforce senescent growth arrest in an autocrine manner (36). Others can stimulate the immune system to clear senescent cells, suppress tumorigenesis, and promote optimal repair of damaged tissues $(15,37)$.

In summary, CD40L-M induces senescence, activates DDR, and inhibits cell proliferation in CD40-positive NSCLC cells. We demonstrated that GATA4 expression is restored by demethylation and triggers $\mathrm{NF}-\kappa \mathrm{B}$ pathway activation to promote senescence in CD40L-M-overexpressing A549/TR cells. This is positively correlated with DDR. Thus, we predict that CD40L-M transgenes may offer an approach to therapeutic intervention via senescence for lung cancer.

\section{Acknowledgements}

The present study was supported by Jiangsu Provincial Key Discipline of Medicine (ZDXKA2016003).

\section{Funding}

The present study was funded by grants from the International Science and Technology Cooperation Program of China (no. 2014DFA31940), the National Natural Science Foundation of China (Beijing, China; nos. 81302014, 81572259 and 81272602), the Six Talent Peaks Project (Jiangsu, China; no. 2015-WSN-038) and the Top Talent Project of 'Six One Engineering' (Jiangsu, China; no. LGY2017071).

\section{Availability of data and materials}

All data generated or analyzed during this study are included in this published article.

\section{Authors' contributions}

JW and WX designed and funded the project. YL, YW, WY, YZ, QH and WZ conducted the experiments and analyzed the data. YL wrote the manuscript. All authors read and approved the manuscript and agree to be accountable for all aspects of the research in ensuring that the accuracy or integrity of any part of the work are appropriately investigated and resolved.

\section{Ethics approval and consent to participate}

The present study does not involve humans, animals and plants.

\section{Consent for publication}

Not applicable.

\section{Competing interests}

The authors declare that they have no competing interests.

\section{References}

1. Cao X, Lai S, Hu F, Li G, Wang G, Luo X, Fu X and Hu J: miR-19a contributes to gefitinib resistance and epithelial mesenchymal transition in non-small cell lung cancer cells by targeting c-Met. Sci Rep 7: 2939, 2017.

2. Hirsch FR, Scagliotti GV, Mulshine JL, Kwon R, Curran WJ Jr, Wu YL and Paz-Ares L: Lung cancer: Current therapies and new targeted treatments. Lancet 389: 299-311, 2017.

3. Wang W, Chen P, Tang M, Li J, Pei Y, Cai S, Zhou X and Chen S: Tumstatin 185-191 increases the sensitivity of non-small cell lung carcinoma cells to cisplatin by blocking proliferation, promoting apoptosis and inhibiting Akt activation. Am J Transl Res 7: 1332-1344, 2015.

4. Ge H, Ni S, Wang X, Xu N, Liu Y, Wang X, Wang L, Song D, Song Y and Bai C: Dexamethasone reduces sensitivity to cisplatin by blunting p53-dependent cellular senescence in non-small cell lung cancer. PLoS One 7: e51821, 2012. 
5. López-Otín C, Blasco MA, Partridge L, Serrano M and Kroemer G: The hallmarks of aging. Cell 153: 1194-1217, 2013.

6. Childs BG, Durik M, Baker DJ and van Deursen JM: Cellular senescence in aging and age-related disease: From mechanisms to therapy. Nat Med 21: 1424-1435, 2015.

7. Kuilman T, Michaloglou C, Mooi WJ and Peeper DS: The essence of senescence. Genes Dev 24: 2463-2479, 2010.

8. Schönbeck U, Mach F and Libby P: CD154 (CD40 ligand). Int J Biochem Cell Biol 32: 687-693, 2000.

9. Gomes EM, Rodrigues MS, Phadke AP, Butcher LD, Starling C, Chen S, Chang D, Hernandez-Alcoceba R, Newman JT, Stone MJ and Tong AW: Antitumor activity of an oncolytic adenoviral-CD40 ligand (CD154) transgene construct in human breast cancer cells. Clin Cancer Res 15: 1317-1325, 2009.

10. Vardouli L, Lindqvist C, Vlahou K, Loskog AS and Eliopoulos AG: Adenovirus delivery of human CD40 ligand gene confers direct therapeutic effects on carcinomas. Cancer Gene Ther 16: 848-860, 2009.

11. Masuda H, Mori M, Uchida T, Uzawa A, Ohtani R and Kuwabara S: Soluble CD40 ligand contributes to blood-brain barrier breakdown and central nervous system inflammation in multiple sclerosis and neuromyelitis optica spectrum disorder. J Neuroimmunol 305: 102-107, 2017.

12. Xu W, Li Y, Wang X, Wang C, Zhao W and Wu J: Anti-tumor activity of gene transfer of the membrane-stable CD40L mutant into lung cancer cells. Int J Oncol 37: 935-941, 2010.

13. Agnihotri S, Wolf A, Munoz DM, Smith CJ, Gajadhar A, Restrepo A, Clarke ID, Fuller GN, Kesari S, Dirks PB, et al: A GATA4-regulated tumor suppressor network represses formation of malignant human astrocytomas. J Exp Med 208: 689-702, 2011.

14. Zheng R and Blobel GA: GATA transcription factors and cancer. Genes Cancer 1: 1178-1188, 2010.

15. Adams PD: Healing and hurting: Molecular mechanisms, functions, and pathologies of cellular senescence. Mol Cell 36: 2-14, 2009.

16. O'Sullivan RJ, Kubicek S, Schreiber SL and Karlseder J: Reduced histone biosynthesis and chromatin changes arising from a damage signal at telomeres. Nat Struct Mol Biol 17: 1218-1225, 2010.

17. Xu W, Xu Y, Wei Y, Tan Y, Zhao H, Zhao W and Wu J: Self-complementary adeno-associated virus 5-mediated gene transduction of a novel CD40L mutant confers direct antitumor effects in lung carcinoma. Mol Med Rep 11: 482-488, 2015.

18. Heo JI, Kim W, Choi KJ, Bae S, Jeong JH and Kim KS: XIAP-associating factor 1, a transcriptional target of BRD7, contributes to endothelial cell senescence. Oncotarget 7: $5118-5130,2016$

19. Fiumara $\mathrm{P}$ and Younes A: CD40 ligand (CD154) and tumour necrosis factor-related apoptosis inducing ligand (Apo-2L) in haematological malignancies. Br J Haematol 113: 265-274, 2001.

20. Wagner AH, Güldenzoph B, Lienenlüke B and Hecker M: CD154/CD40-mediated expression of CD154 in endothelial cells: Consequences for endothelial cell-monocyte interaction. Arterioscler Thromb Vasc Biol 24: 715-720, 2004

21. Srahna M, Remacle JE, Annamalai K, Pype S, Huylebroeck D, Boogaerts MA and Vandenberghe P: NF-kappaB is involved in the regulation of CD154 (CD40 ligand) expression in primary human T cells. Clin Exp Immunol 125: 229-236, 2001.

22. Durie FH, Foy TM, Masters SR, Laman JD and Noelle RJ: The role of CD40 in the regulation of humoral and cell-mediated immunity. Immunol Today 15: 406-411, 1994.

23. Loskog A, Maleka A, Mangsbo S, Svensson E, Lundberg C, Nilsson A, Krause J, Agnarsdóttir M, Sundin A, Ahlström H, et al: Immunostimulatory AdCD40L gene therapy combined with low-dose cyclophosphamide in metastatic melanoma patients. $\mathrm{Br}$ J Cancer 114: 872-880, 2016.
24. Beatty GL,Chiorean EG,Fishman MP, Saboury B, Teitelbaum UR, Sun W, Huhn RD, Song W, Li D, Sharp LL, et al: CD40 agonists alter tumor stroma and show efficacy against pancreatic carcinoma in mice and humans. Science 331: 1612-1616, 2011.

25. Elmetwali T, Young LS and Palmer DH: CD40 ligand-induced carcinoma cell death: A balance between activation of TNFR-associated factor (TRAF) 3-dependent death signals and suppression of TRAF6-dependent survival signals. J Immunol 184: 1111-1120, 2010.

26. Campisi J: Cellular senescence: Putting the paradoxes in perspective. Curr Opin Genet Dev 21: 107-112, 2011

27. Pazolli E, Alspach E, Milczarek A, Prior J, Piwnica-Worms D and Stewart SA: Chromatin remodeling underlies the senescence-associated secretory phenotype of tumor stromal fibroblasts that supports cancer progression. Cancer Res 72 : 2251-2261, 2012.

28. Liu Y, Hawkins OE, Su Y, Vilgelm AE, Sobolik T, Thu YM, Kantrow S, Splittgerber RC, Short S, Amiri KI, et al: Targeting aurora kinases limits tumour growth through DNA damage-mediated senescence and blockade of NF- $\kappa \mathrm{B}$ impairs this drug-induced senescence. EMBO Mol Med 5: 149-166, 2013.

29. Toledo LI, Murga M, Gutierrez-Martinez P, Soria R and Fernandez-Capetillo O: ATR signaling can drive cells into senescence in the absence of DNA breaks. Genes Dev 22: 297-302, 2008

30. Gire V, Roux P, Wynford-Thomas D, Brondello JM and Dulic V: DNA damage checkpoint kinase Chk2 triggers replicative senescence. EMBO J 23: 2554-2563, 2004.

31. Azhikina T, Kozlova A, Skvortsov T and Sverdlov E: Heterogeneity and degree of TIMP4, GATA4, SOX18, and $E G F L 7$ gene promoter methylation in non-small cell lung cancer and surrounding tissues. Cancer Genet 204: 492-500, 2011.

32. Campisi J: Aging, cellular senescence, and cancer. Annu Rev Physiol 75: 685-705, 2013

33. Kang C, Xu Q, Martin TD, Li MZ, Demaria M, Aron L, Lu T, Yankner BA, Campisi J and Elledge SJ: The DNA damage response induces inflammation and senescence by inhibiting autophagy of GATA4. Science 349: aaa5612, 2015.

34. Freund A, Patil CK and Campisi J: p38MAPK is a novel DNA damage response-independent regulator of the senescence-associated secretory phenotype. EMBO J 30: 1536-1548, 2011.

35. Rovillain E, Mansfield L, Caetano C, Alvarez-Fernandez M, Caballero OL, Medema RH, Hummerich $\mathrm{H}$ and Jat PS: Activation of nuclear factor-kappa B signalling promotes cellular senescence. Oncogene 30: 2356-2366, 2011.

36. Acosta JC, Banito A, Wuestefeld T, Georgilis A, Janich P, Morton JP, Athineos D, Kang TW, Lasitschka F, Andrulis M, et al: A complex secretory program orchestrated by the inflammasome controls paracrine senescence. Nat Cell Biol 15: 978-990, 2013

37. Kang TW, Yevsa T, Woller N, Hoenicke L, Wuestefeld T, Dauch D, Hohmeyer A, Gereke M, Rudalska R, Potapova A, et al: Senescence surveillance of pre-malignant hepatocytes limits liver cancer development. Nature 479: 547-551, 2011.

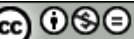

This work is licensed under a Creative Commons Attribution-NonCommercial-NoDerivatives 4.0 International (CC BY-NC-ND 4.0) License. 